\title{
Participatory Design of a Cooperative Exploration Mediation Tool for Human Deep Space Risk Mitigation
}

\author{
Donald Platt ${ }^{1}$. Patrick Millot ${ }^{2}$, and Guy Andre Boy ${ }^{1}$ \\ ${ }^{1}$ Human-Centered Design Institute, Florida Institute of Technology, \\ Melbourne, FL 32901, USA \\ \{dplatt, gboy\} @fit.edu \\ ${ }^{2}$ LAMIH CNRS, University of Valenciennes, France \\ patrick.millot@univ-valenciennes.fr
}

\begin{abstract}
This work describes the participatory design and development of a Virtual Camera (VC) system to improve astronaut and mission operations exploration efficiency and cooperation while exploring in deep space. Advanced interaction media capabilities can improve exploration efficiency and cooperation as the distribution of human space exploration roles change in deep space exploration. This capability was developed in a tablet-based application that was evaluated in the field. The VC can minimize the risk of astronauts exploring unknown reaches of the solar system with limited previous knowledge of the area under exploration. Ground-based expert knowledge can be captured and be easily assessable in the remote deep space environment with the VC. The human-centered method of development and testing is described as well as results.
\end{abstract}

Keywords: Situation Awareness (SA);Augmented Reality; Human-Computer Interaction (HCI); Tablet Computing; Usability Testing; Space Exploration.

\section{Introduction}

The Virtual Camera (VC) is not a specific interface or a single platform. It is a database exploration concept providing the ability to interact with the database with multiple interaction methods. The VC concept is an agent to assist in enhancing future space exploration. With the orchestra organizational model [1] all actors are acting with the same sheet of music or are on the same page. As organizations progress in the 21 st century they are no longer well suited for a hierarchical structure. Roles continue to change and be redefined, much like how the roles and emphasis changes in a complex piece of music. Human deep space exploration is facing this challenge. The VC is designed to help in this role re-assignment process. Goals, such as areas to be explored, can be shared among team members so that everyone is aware of what should be explored, what team members are currently exploring and also what areas should be avoided.

There is a continuous human-human interaction in space exploration that will be limited due to communication delays caused by distance in deep space exploration. The 
control model is switching from one of supervision from the Earth-based controllers to one of mediation between the ground and the astronauts, see Figure 1. Risk will be increased as astronauts roles change to one of more autonomous decision making as opposed to ground-mediated decision making. Risks such as decision making based upon incomplete information or a lack of domain knowledge are very real for astronauts exploring in deep space. Real-time decision making will be required of astronauts without real-time support from operators back in mission control due to the communications delays in deep space. Tools will be required that support the astronaut's knowledge-based reasoning and abduction to collaborate with human judgment.

An important aspect of the VC is the idea of improved team or multi-agent situation awareness for exploration. This takes into account the goals and requirements of each individual user. Rather than being concerned that everyone involved understands the same thing, the $\mathrm{VC}$ is designed and used from the perspective of understanding how each user sees the exploration goal from their own unique perspective. This is much like the musicians in an orchestra. They all have their own instruments and parts of the music, but they need to play together to make art coordinated by a conductor. Human deep space missions will be characterized by remote distributed operations. There will be a need to make decisions based upon the collection and analysis of raw data to provide predictive information. This information needs to be presented to crews in a way that enhances situation awareness.

The Virtual Camera (VC) is an interactive 3-D tablet device that allows astronauts to capture expert knowledge about the areas they will explore [2]. The VC integrates and manages mission data and areas of interest for both exploration and safety. This information will include system health and status, caution and warning, safety, traverse execution and mission timeline parameters. This paper explores the VC and these changing roles for deep space human exploration and the new human-machine cooperation needed for deep space exploration and how this system can mitigate risk in human deep space exploration. Testing and evaluation explore how the VC influences interaction and cooperation between actors involved in human spaceflight.

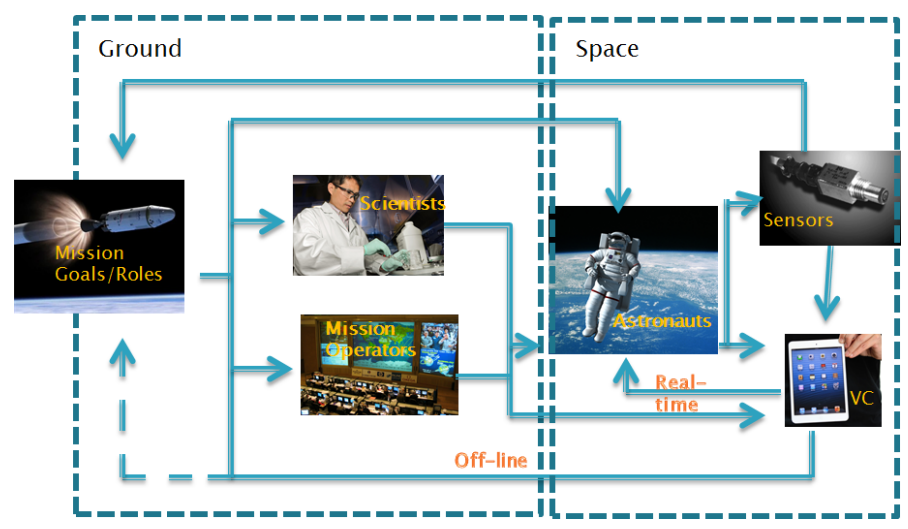

Fig. 1. The model of the virtual camera interaction with a space segment, used by astronauts and a ground segment used by mission operators and scientists 


\section{Motivation}

Interactive cockpits, context-sensitive systems and remote agent knowledge representation are being developed for a number of domains. Modern users of computer technology are very familiar with and aware of tablet-based interaction technology. This technology should also be applied to the next generation of human space exploration systems. The VC demonstrates these possibilities in a system usable for deep space human exploration.

Astronauts were familiar with relatively simple analog and CRT displays in the era of the space shuttle. This is now changing with new "interactive" cockpits. Older astronauts will be less familiar with the concept of interactive cockpits but younger ones will most likely have used them for most of their flying careers. Tablet and other advanced interaction media systems are now commonplace in a number of domains including home usage and stakeholders for the VC will have exposure to this type of interaction making it a natural for future space exploration systems.

Mission operations personnel will be interested in using the VC for mission planning purposes as well as training. For the actual mission the large distances involved between Earth and the body being explored means that communications delays will make it impossible to provide real-time feedback to astronauts. This requires the VC to capture the expertise of the mission operations personnel on-board. The goal is to make the VC a remote assistant/agent for the mission operations personnel.

Scientists will also use the VC for planning future traverses and to look for new discoveries on the body being explored. Their experience level with the VC tool will typically be less than the astronauts and mission operations personnel. They will want to denote and annotate areas of scientific and exploration interest to them. This information can be archived late retrieved by other VC users. The virtual camera can provide a third-person perspective to assist in the navigation process for rover and other exploration vehicles. However, it is more than just a "back-up" camera that is now provided on many terrestrial automobiles.

The VC was implemented on a portable tablet computer offers many unique advantages including the ability to bring the device around the cockpit to any required vantage point to explore the remote planetary surface. For instance, a crew member using a tablet-based VC can carry it easily from one window to the next as the vehicle maneuvers around areas of interest. It can also carry electronic procedures that can be pulled-up and displayed depending upon the situation. The VC can also be carried by outside explorers who are walking across the surface of the planetary body. Most of the testing presented in this work was completed in this scenario, with users carrying the device while navigating and exploring on foot. Other possible uses and configurations include using the device in a planning scenario in conjunction with large format and three-dimensional displays on earth or at a habitation base. A portable, tablet device allows improved interaction, collaboration and sharing of new knowledge to improve efficiency of exploration. In this case, efficiency is measured by the ability to quickly identify areas of interest to explore and then to explore more areas in a given period of time. Greater situational awareness leads to higher levels of efficiency because additional areas can be explored, i.e. more knowledge can be gained (and 
retained) on a single expedition, thereby reducing the number of expeditions required. It provides one integrated tool for navigation and exploration assistance as well as annotation.

\section{Related Work}

Endsley [3] broke situation awareness down into three elements, perception, comprehension, projection. The VC contributes to improving exploration situation awareness on all three levels, see Figure 2. Perception is improved by offering multiple views and perspectives of the scene around the user. This could be correlated with vehicle position data to show a real-time situation or it can be used as a training element showing where a vehicle may be at a certain time during a traverse.

Using the VC for deep space exploration, comprehension is improved by allowing the user to understand more about their environment than what is possible just by looking out of the window of the vehicle. With an annotation capability knowledge from domain experts can also be incorporated into the database. For instance, mission operations people can annotate areas of particular safety or science interest during training and these notes and other information can then be pulled up when the astronaut is actually at that location on the remote planet.

Future deep space human exploration will require a transfer of function allocation from Earth-based mission control to on-board the spacecraft. Communications delays due to distance will require crews to be more autonomous and not rely on mission control for real-time advice and control. For example, the time delay in communications to the moon will take 6 seconds round trip, and up to 44 minutes for Mars. For missions lasting perhaps more than a year training conducted on the ground may be forgotten. Skill-retention issues will be a concern. On long-duration space station missions today, astronauts often find they are pulling information from memory in what becomes a subconscious effort. Training can take place months before the actual space-based operation takes place. This capability to pull from memory the skills needed to complete the mission may be diminished when practice is no longer possible during life-critical deep space mission. On-board support and refresher training will be required. The $\mathrm{VC}$ allows an astronaut to move through a traverse or exploration sequence ahead of time. Tools are currently being tested to capture mission control knowledge and expertise on-board a deep space exploration vehicle.

Projection will be improved by allowing traverses to be rehearsed ahead of time. The best route can be determined and planned. Safety and exploration performance considerations can be taken into account. Possible future actions can be modelled and best choices made. Millot and Hoc [4] defined two concepts in which cooperation between humans and machines takes place, the first is know-how and the second is know-how-to cooperate. Know-how is the abilities or information of a single agent. The latter involves agent-to-agent interaction. In the case of the VC the know-how is being transferred from ground personnel to astronauts through a mediation agent, the VC. This provides a common work space for the interaction. 
Dynamic allocation of tasks will be required in human deep space exploration. Mission control, which may then be called mission support in this case, may be able to make some mission decisions but oftentimes the astronauts will be required to do so on their own. Pacaux-Lemoine and,Debernard [5] broke down human-human agent cooperation during possible decision interference in three ways, negotiation, acceptation and imposition. For the VC interaction, negotiation involves improving the frame of reference of ground personnel and astronauts and exploration goals. Certain mission goals may take priority at different times and the VC can help this prioritization. Acceptation will involve agreement on the next exploration course of action. Finally, imposition will require defining next course of action without negotiation in some cases when for instance communication blackouts or emergencies cause a decision to have to be made without the ability to interact at all with Earth.

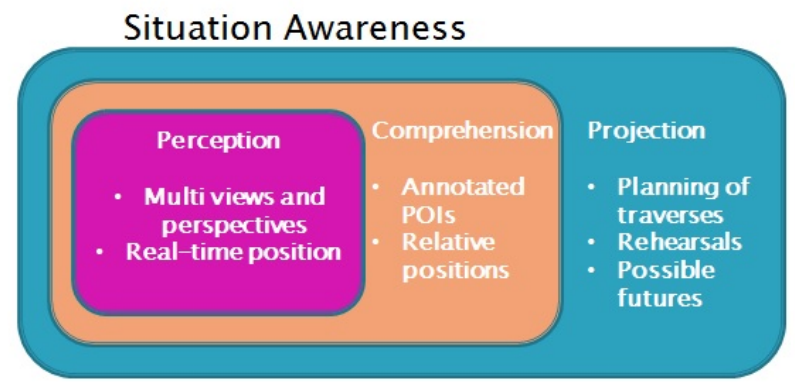

Fig. 2. The VC allows improved exploration situation awareness on the levels of perception, comprehension and projection

Stanton has suggested that teams cannot truly share situation awareness [6]. In a collaborative environment such deep space exploration we need to be concerned with distributed cognition where appropriate information, held by individuals but captured by devices represents s dynamically changing environment. The VC must transfer this appropriate information to appropriate team members at the appropriate time.

Collaboration can be mediated through computer systems in what is known as computer supported collaborative work (CSCW). Collaboration can involve a few individuals or a team. CSCW is concerned with how technology can effect collaboration [7]. Grudin and Poltrock also looked at human behaviors that contribute to collaboration and broke them down into three main categories: communication, sharing of information and coordination. Collaboration can be real-time or asynchronous. Systems that enable or enhance collaboration should enhance these behaviors in real-time or asynchronously. Modern social media technologies such as Twitter and Facebook combine communications and information sharing capabilities. Social units are also important, such as small groups, teams, projects, organizations and communities.

The VC encourages user interaction within its database. According to Grudin and Poltrock, determining how to route, organize, and present contextual information to facilitate collaboration is a pressing challenge. They also suggested that qualitative 
field research is an area with unlimited potential. New technology deployment always provides phenomena of interest. Qualitative research can find patterns that are then followed up with qualitative research to discover what the patterns mean. Collaboration and collaborative exploration bring to mind teams and the concept of team situation awareness. There has been considerable debate about the usefulness of team situation awareness and even how to measure it [6]. Team members may have different immediate goals and certainly different roles within the team environment. Therefore, it may be a detriment for all team members to share the same situation awareness [8]. In fact, the cognitive properties of a group may differ from the individuals who make up the group [9]. Even for analyzing individual's situation awareness, it is difficult to apply probes such as Endsley's SAGAT during real-world collaborative tasks [6]. Reality often involves events happening in parallel and serial models do not do well in accounting for this.

\section{Participatory Design}

A human-centered design approach has been used to develop the tablet-based VC. The first step was to develop low-level prototypes to capture the basic design requirements for the VC. The initial point in determining user requirements is to identify experts that represent all possible user types for the VC system. This requires some knowledge of the domain and also of the goals of the VC. A survey of potential users and stakeholders (astronauts, mission operations personnel, and scientists) for the VC system was conducted by the authors at the 2011 NASA DesertRATS analog exploration testbed. The main questions during potential user interviews involved their background, general system uses, interface type and data display parameter formats desired.

Brief scenarios and interaction diagrams as well as story-boards and prototypes have also been developed. These can be presented to users to show the basic concept of the $\mathrm{VC}$ and to get feedback of what would be a beneficial inter-face for a particular user and what may also not be of benefit to a user. Scenarios and use cases give users examples of how the VC can be applied. Next horizontal prototypes were developed that captured the basic interaction requirements for the VC and were used to demonstrate an operational scenario.

A complete vertical prototype was then developed with all of the interaction capability using a tablet PC device. Usability of the VC will be evaluated using nominal and off-nominal situations and simulations of actual mission operations cases. Important considerations include what is appropriate for a given task or function in a given context such as after an alarm or interruption versus normal operations.

Usage of the vertical prototype determined how unique aspects of the tablet improve or impact situation awareness, exploration efficiency and collaborate.. The completed tablet $\mathrm{VC}$ is a tool that is used to analyse ways to improve situation awareness as well as illustrate how interactions between collaborators and their various roles change in deep space exploration compared to low-earth orbit (LEO) operations. 
Usage of the system in the field also illustrated other applications for the VC beyond space exploration as well as emergent behaviors.

\section{Evaluation}

Preliminary testing of the VC has been completed to identify the interaction capabilities and issues when operating in deep space Initial tests have been conducted in a terrestrial setting (a public park) with areas of interest (AOIs) defined to be of science or resource use as well as hazards. These points have been entered into the VC Google map terrain database. After lessons learned in the first set of evaluations, a second set were conducted with a team of scientists in a true science exploration setting.

For the first round of testing three teams were involved with 8 different subjects in each who all had tablet experience. The goal was not to gather a large statistical sample but rather to gather feedback and collect emergent behaviors and uses of the system. The subject team used the VC to navigate a course encountering AOIs of various point values to simulate mission importance scale. The order of AOIs was defined by a Mission Control (MC) team ahead of time in consultation with the VC subject team. A control team will also be on the same course using a non-interactive tablet-based map of the area. The control team used a feature limited version of Virtual Camera which can display static AOIs and report data to MC. The MC team had access to the same database of AOIs as the Subject and Control teams, although communication between the teams in the field and MC team involved a simulated lightspeed delay. The communication delay was done by having a phone in the possession of the observer of each of the two field teams who did NOT answer the phone. The MC team left voice mail responses. The observer waited an agreed upon time delay, then played back the voicemail. Thus half duplex, time delayed messaging was simulated. This simulated the changing environment the $\mathrm{VC}$ is expected to operate in. Pictures were taken by the field teams to confirm that each resource site was visited correctly.

Observations were made of the test subjects in the field. They were asked to speak aloud and give their impressions of the use of the VC or map-only interface to assist them in determining what to explore. Questionnaires were used to evaluate navigation situation awareness during VC testing and to compare it to teams in the field who had the map only for navigation. For navigation situation awareness, at two points in each 30 minute traverse both the VC and map only subjects were asked to answer a series of questions about their ability to perceive their situation, comprehend it and project into the future. The observer then determined whether the answer was appropriate or not for the given situation. This situation awareness assessment tool is referred to in this study as the Knowledge-Based Situation Assessment Tool (KB-SAT) and its characteristics are:

- Develop a series of questions to be asked of users in the field

- questions should probe at one of the three stages of situation awareness formation (perception, comprehension and projection) 
- Give a brief (30 second) period for the subject to respond, using the device being evaluated (VC)

- Log whether the response was appropriate or not ( for example: was the nearest object of interest identified correctly?)

Questionnaires based upon the KB-SAT situation awareness level protocol as well as the NASA TLX workload index, the SUS Likert scale and the Modified CooperHarper tool were given the teams.

The subject teams using the full VC interaction system found themselves relying more heavily on the $\mathrm{VC}$ tablet application to the point that when the wireless link to the Google database was lost the team felt somewhat confused about their location. This shows the need for data validation and a thorough review of data in the VC by system experts before and after each traverse or sortie. In the future, icons could also change color or intensity based upon confidence level of data entered into the database.

Debriefings were conducted of teams after their in-field evaluations. They were asked to rate their confidence (trust) in navigation, location finding and identifying exact position of themselves and AOIs/hazards in the field with either the map only or the VC. They would select a value from 1 to 5 , with 1 being no confidence and 5 being complete confidence. Using feedback from these debriefings, it was determined that using the map alone with none of the interactive capability of the VC, the control team found they had high confidence in where in the course they were but lower confidence on what exact AOIs they were looking for, see Table 1. They did not have the ability to see the AOIs marked off at a higher zoom-in level and had no access to the annotation information placed by the simulated ground experts in the database. They found themselves surveying actual landmarks often to get their bearings. This indicates a reliance on the map for navigation but more unknowns about the actual AOIs and what the team was actually looking for. Since time is precious during space-based exploration, efficiency and precision are very important.

VC users were operating in a much more "heads-down" mode relying more heavily on the technology. The breadcrumb display was useful for finding where the team was and the direction they were headed, much more useful than the large heading arrow. The $\mathrm{VC}$ teams felt with a high level of confidence where they were headed and where the AOIs were. This indicates the VC is quite useful for defining, identifying and locating the AOIs.

Table 1. Confidence ratings (scale 1 to 5) for navigation and AOI identification for both VC and map only (averages and standard deviation)

\begin{tabular}{|c|c|c|c|c|}
\hline & $\begin{array}{c}\text { VC Naviga- } \\
\text { tion }\end{array}$ & $\begin{array}{c}\text { VC AOI Identi- } \\
\text { fication }\end{array}$ & $\begin{array}{c}\text { Map Only } \\
\text { Navigation }\end{array}$ & $\begin{array}{c}\text { Map Only } \\
\text { AOI Identifica- } \\
\text { tion }\end{array}$ \\
\hline Average & 4.4 & 4.0 & 3.4 & 3.0 \\
\hline Std Dev & 0.79 & 0.58 & 0.55 & 0.71 \\
\hline
\end{tabular}


Tests were conducted with a simulated communications delay between the field teams and mission control. This was done by having an observer communicate by text message with mission control in order to answer questions. The mission control team would then wait a predetermined amount of time and then respond. It was observed that the VC field teams would often not wait for responses to questions that they texted to mission control, especially considering the 30 minute traverse time limit. They would use the $\mathrm{VC}$ and their own knowledge to make decisions and begin improvising and completing the action without waiting for a response and move forward with their exploration and search for the defined areas of interest. When the team did receive the mission control text response they would use the response for confirmation of further action, relying on their own decision-making capability. The large risk associated with the time delay between mission control and astronauts in deep space exploration will require local tools that capture some of the remote knowledge and expertise. This highlights that the VC needs to enable the interface that makes the recall and interpretation of this captured knowledge as seamless as possible. Also the VC enables a closer collaboration and sharing of information between several astronaut teams who may be exploring in the field. The explorers can add information about sites of interest and scientifically important items as they are uncovered in the field. This also illustrates the changes in collaboration and cooperation for deep space missions. To expedite the exploration process astronauts far from Earth will not be able to wait for responses from Earth and require on-board decision making assistants such as the VC. They will be switching between what Hollnagel [Hollnagel, 1998] and Stanton [Stanton, et al, 2008] described as phenotype and genotype Schemas. They may revert back to training or previous knowledge if the $\mathrm{VC}$ is not operational.

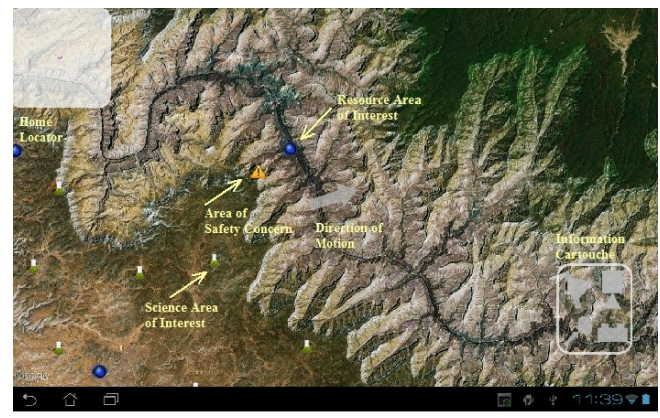

Fig. 3. The virtual camera interaction system showing the points of interest and information display

To determine the possibilities offered by the VC and to evaluate its use in an exploration environment, the VC was used at an astrobiology scientific expedition involving a science team led by Dr. Chris McKay of the NASA Ames Research Center and a group of teachers from around the state of Idaho. The evaluation was conducted as part of the Spaceward Bound summer exploration session at Craters of the 
Moon national monument in Idaho sponsored by the NASA Idaho Space grant consortium. This unique park, with lava flows and volcanoes similar to those found on the moon, was used to train the Apollo era astronauts before they went to the moon.

Astrobiologists and geologists from NASA Ames Research Center, Idaho State, Idaho University and teachers mapped the lichen growth at a variety of places around the Craters of the Moon national park using the VC as an assistant. One goal was to compare the ability to conduct field science with traditional tools of a digital camera, GPS device and field notebook to using one device, the VC. Also, collaboration using the VC to map scientifically important objectives was observed. Three days of field use studies were obtained while the team mapped geologic and biological (moss, lichens) components in lava flows. Overall, the VC was evaluated for science utility, collaboration and use for education.

Evaluation was conducted by training the scientists on the use of the system and then allowing them to take it into the field to use it during their field studies, see Figure 4. A goal was to make the use of the VC as noninvasive as possible for the work they were conducting. Part of the goal of the expedition was to use a hand-held x-ray fluorescent spectrometer to map the concentration of elements in the rocks around lichen outcroppings. This device produced a read-out of the elemental concentrations. The VC was then used to capture the element compositions and these were then combined with a picture taken of the site showing where the data was collected and other scientific notes about the sample. The VC then provided a geo-located scientific record of the measurement including observations and other documentation, see Figure 5. This figure shows the record as seen in the web page database that can be called up from any computer on the internet, showing the exact location the sample was taken.

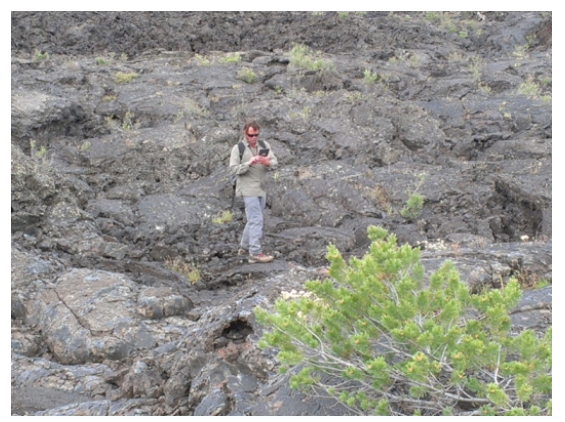

Fig. 4. A scientist using the VC to collect and annotate science data in the field

The points of interest, of course, can also be pulled up within the VC tablet interface as well. Using the VC interface points in the database can be viewed or edited. Additional information can be added as well. So, before or during a traverse explorers can pull up the information and see what points are nearby and what data is available on them. For the field tests, where it should be noted the scientists were evaluating the device on their own, they rated the VC about the same as current systems for interaction and facilitating scientific discovery. The encouraging results are 
that overall the VC rates very favorably for scientific exploration for these scientists and in no evaluation point did it rate worse than current systems. Conflict resolution and finding points to explore faster were the two areas with the highest comparative rating for the VC. This is encouraging as well for a device intended to be used as a decision support aid and improvisation tool when outside expertise may not be available.

Several different types of data were gathered such as, situational awareness, VC tablet utilization, overall success of mission. A web application was the interface between the database and the Mission Control team. It allowed MC to observe breadcrumbs of the teams and observe how well they were fairing in making it to the desired AOIs.

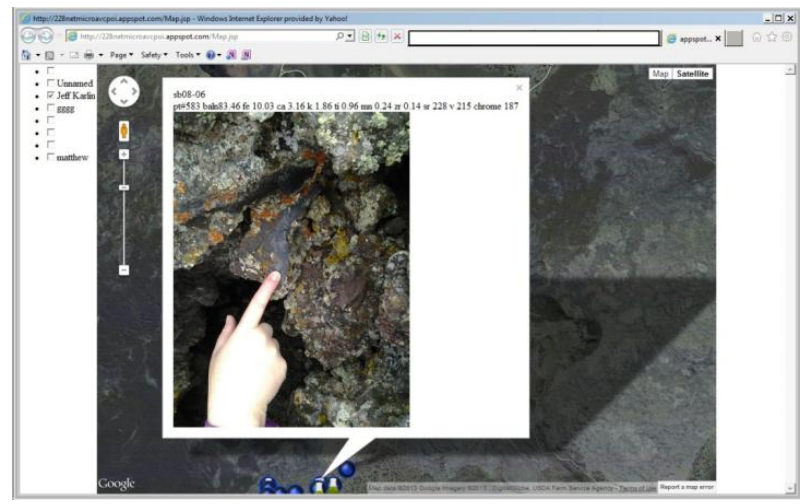

Fig. 5. A science data point entered into the VC database showing the annotation, data collected by a hand held sensor and pointing to the spot on the rock that the data was collected. View is of the web page data center.

\section{Conclusions}

The virtual camera for planetary exploration will assist in future exploration involving humans and robots. It is designed to provide improved exploration situational awareness and collaboration to define areas of interest and safety concern on remote planetary bodies. It acts as remote agents for mission planners and scientists capturing their knowledge and expertise as astronauts explore the solar system.

Human-centered design techniques are being applied to the development of this tool involving all potential user groups from the beginning of the design process. Prototypes have been developed and several scenarios considered and evaluated in the field.

Moving forward, the VC interaction and incrementally updated database technology can be applied to other domains such as law enforcement, disaster first responders, aviation cockpit weather displays and control room visualizations. 


\section{References}

1. Boy, G.A.: Orchestrating Human Centered Design. Springer, London (2013)

2. Platt, D., Boy, G.A.: The Development of a Virtual Camera System for Astronaut-Rover Planetary Exploration. In: Proceedings of the 2012 IEA World Congress on Ergonomics, Recife, Brazil (2012), doi:10.3233/WOR-2012-0032-4532

3. Endsley, M.: Towards a Theory of Situation Awareness in Dynamic Systems. Human Factors 37(1), 32-64 (1995)

4. Millot, P., Hoc, J.M.: Human-machine cooperation: Metaphor or possible reality? In: Proceedings of ECCS 1997, Manchester, April 9-11 (1997)

5. Pacaux-Lemoine, M.P., Debernard, S.: Common work space for human-machine cooperation in air traffic control. Control Engineering Practice 10, 571-576 (2002)

6. Stanton, N., Salmon, P., Walker, G., Jenkins, D.: Genotype and phenotype schemata and their role in distributed situation awareness in collaborative systems. Theoretical Issues in Ergonomics Science 10(1), 43-68 (2009)

7. Grudin, J., Poltrock, S.: Computer Supported Cooperative Work. In: Soegaard, M., Dam, R.F. (eds.) The Encyclopedia of Human-Computer Interaction, 2nd edn. The Interaction Design Foundation, Aarhus (2013)

8. Gorman, J.C., Cooke, N., Winner, J.L.: Measuring team situation awareness in decentralised command and control environments. Ergonomics 49, 1312-1326 (2006)

9. Hutchins, E.: Cognition in the wild. MIT Press, Cambridge (1995) 